\title{
Clinical Study of Insulin Glargine in the Treatment of Elderly Patients with Type 2 Diabetes Mellitus Complicated with Critical Medical Patients
}

\author{
Xin Wang, Yuanyuan Zhang* \\ Department of Endocrinology, Shaanxi Provincial People’s Hospital, 256 Youyi West Road, Xi’an, China.
}

\begin{abstract}
How to cite this paper: Xin Wang, Yuanyuan Zhang. (2021) Clinical Study of Insulin Glargine in the Treatment of Elderly Patients with Type 2 Diabetes Mellitus Complicated with Critical Medical Patients. International Journal of Clinical and Experimental Medicine Research, 5(3), 344-348.

DOI: 10.26855/ijcemr.2021.07.017
\end{abstract}

Received: May 28, 2021

Accepted: June 20, 2021

Published: June 28, 2021

*Corresponding author: Yuanyuan Zhang, Department of Endocrinology, Shaanxi Provincial People’s Hospital, 256 Youyi West Road, Xi’an, China.

\begin{abstract}
Objective: To study the clinical effect of insulin glargine in the treatment of elderly patients with type 2 diabetes mellitus complicated with critical medical patients. Methods: 100 elderly patients with type 2 diabetes mellitus complicated with medical critical illness in our hospital from February 2020 to February 2021 were selected retrospectively. According to the treatment methods, they were divided into two groups: neutral low protamine zinc human insulin single treatment group (single treatment group), neutral low protamine zinc human insulin combined with insulin glargine treatment group (combined treatment group), 50 cases in each group blood glucose level, serum C-peptide level and incidence of hypoglycemia. Results: The blood glucose concentration of the combined treatment group was lower than that of the single treatment group on 1D, 3D and 5D after treatment $(P<0.05)$, but there was no significant difference between the two groups before treatment, $7 \mathrm{~d}$ and $10 \mathrm{~d}$ after treatment $(P>0.05)$. After treatment, the fasting blood glucose, $2 \mathrm{~h}$ postprandial blood glucose and glycosylated hemoglobin of the combined treatment group were lower than those of the single treatment group $(P<$ 0.05 ). After treatment, the levels of fasting C-peptide and postprandial $2 \mathrm{H}$ $\mathrm{C}$-peptide in the combined treatment group were higher than those in the single treatment group $(P<0.05)$. The incidence of hypoglycemia in the combined treatment group was $10.00 \%(5 / 50)$, which was lower than $52.00 \%$ $(26 / 50)$ in the single treatment group $(P<0.05)$. Conclusion: The clinical effect of insulin glargine in the treatment of elderly patients with type 2 diabetes mellitus complicated with critical medical patients is good.
\end{abstract}

\section{Keywords}

Insulin glargine, Neutral low protamine zinc, human insulin, Old age, Type 2 diabetes mellitus, Critically ill in internal medicine, Blood glucose concentration, Blood glucose level, Serum c-fetoprotein level, hypoglycemia

For the elderly critical patients, due to the reverse regulation of the activation of sex hormones and the release of cytokines in the stress state, the increase of blood glucose is greater than the regulation level of insulin, which leads to the occurrence of hyperglycemia. At present, many studies have shown that strict control of blood glucose levels in elderly critically ill patients can effectively improve the prognosis of patients [1]. Therefore, the American Diabetes Association and the Endocrine Society issued a consensus document on blood glucose control in hospitalized patients in August 2006 [2]. The document points out that the blood glucose level of the elderly patients with criti- 
cal illness should be controlled as soon as possible and timely, and insulin is the first choice when the blood glucose level is strictly controlled. Because insulin can not only effectively control the blood glucose level of patients, but also improve the metabolic disorders, inflammatory response and blood coagulation of patients. In addition, insulin therapy can effectively inhibit the activity of NF-kB and increase the expression of anti-inflammatory cytokines, such as IL-2 and IL-4. At the same time, it can reduce the expression of ICAM-1 and MCP-1, effectively prevent leukocyte aggregation and adhesion to endothelial cells in hemostatic circulation, improve vascular endothelial function and inhibit thrombosis [3]. Therefore, timely use of insulin therapy can effectively reduce the mortality of patients. This paper studies the clinical effect of insulin glargine in the treatment of elderly patients with type 2 diabetes mellitus complicated with critical medical patients.

\section{Materials and methods}

\subsection{General information}

A total of 100 elderly patients with type 2 diabetes mellitus complicated with medical critical illness in our hospital from February 2020 to February 2021 were selected retrospectively. According to the treatment methods, they were divided into two groups: neutral low protamine zinc human insulin single treatment group (single treatment group), neutral low protamine zinc human insulin combined with insulin glargine treatment group (combined treatment group). The age of 50 patients in the combined treatment group was 62-82 years old, with an average of (72.45 4.6\%) \pm There were 22 females (44.00\%) and 28 males (56.00\%). In terms of course distribution, 28 cases (56.00\%) were in 1-10 years and 22 cases (44.00\%) were in 11-20 years; In terms of the types of critical medical diseases, there were 9 cases $(18.00 \%)$ of tumor chemotherapy, 9 cases $(18.00 \%)$ of severe pneumonia, 8 cases $(16.00 \%)$ of severe acute pancreatitis, 8 cases $(16.00 \%)$ of chronic heart failure, 6 cases $(12.00 \%)$ of acute coronary syndrome, 5 cases $(10.00 \%)$ of uremia hemodialysis and 5 cases $(10.00 \%)$ of respiratory failure. The age of 50 patients in the single treatment group ranged from 63 to 83 years old, with an average of (73.52 $1 \pm$ ). There were 21 females $(42.00 \%)$ and 29 males $(58.00 \%)$. In terms of course distribution, 27 cases $(54.00 \%)$ were in 1-10 years and 23 cases $(46.00 \%)$ were in 11-20 years; In terms of the types of critical medical diseases, there were 10 cases (20.00\%) of tumor chemotherapy, 10 cases $(20.00 \%)$ of severe pneumonia, 9 cases $(18.00 \%)$ of severe acute pancreatitis, 7 cases $(14.00 \%)$ of chronic heart failure, 5 cases $(10.00 \%)$ of acute coronary syndrome, 5 cases $(10.00 \%)$ of uremia hemodialysis and 4 cases $(8.00 \%)$ of respiratory failure. There was no significant difference in general information between the two groups $(\mathrm{P}>0.05)$. This study was approved by the medical ethics committee of our hospital, and all patients had informed consent.

\subsection{Inclusion and exclusion criteria}

Inclusive criteria: 1) all patients had complete medical records; 2) All of them met the diagnostic criteria of type 2 diabetes [4]; 3) They all had good compliance. Exclusion criteria: 1) coagulation dysfunction; 2) Combined with immune system diseases; 3) Combined with hematological diseases.

\subsection{Methods}

\subsubsection{Single treatment group}

The patients were treated with human insulin of neutral low protamine zinc, 0.2-0.4 U/kg, once a night, subcutaneous injection. For the patients who can eat, short acting insulin was added to the patients before meals, the dose was $0.13 \mathrm{U} / \mathrm{kg}$, and the blood glucose concentration was controlled in the range of 5.0-8.0 mmol/L. When the fasting blood glucose concentration was more than $8.0 \mathrm{mmol} / \mathrm{L}$, the dose of insulin needed to be corrected. According to the estimated insulin sensitivity, the level of corrected dose was 1-4 u for every $3.0 \mathrm{mmol} / \mathrm{L}$ increase of blood glucose level. Every 1-2 days, 2-6 u basal insulin dose was adjusted to make the blood glucose reach the target value. If the curative effect is not ideal, adjust the corrected insulin dose once every 1-2 days, and adjust 1-2 U/time for each grade.

\subsubsection{Combined treatment group}

On the basis of the single treatment group, the patients were treated with insulin glargine, 0.2-0.4 U/kg, once a night, subcutaneous injection.

The two groups were treated for 10 days. The goal of blood glucose control: 5.0-8.0 mmol/l. The blood glucose of fingertips was detected by blood glucose meter. The blood glucose was detected 5 times a day, which was before three meals and 9:00 p.m. and 2 a.m. The average blood glucose of the day was calculated. For patients with hypoglycemia, they need to be tested. If the patient has hypoglycemia, the insulin amount of the first day $\times 80.0 \%$ to 
adjust the insulin dosage.

\subsection{Observation index}

The patients were followed up for 1 week. 1) Blood glucose concentration; 2) Blood glucose level. Fasting blood glucose, $2 \mathrm{~h}$ postprandial blood glucose and glycosylated hemoglobin were included. The standard of blood glucose: fasting blood glucose $<7.8 \mathrm{mmol} / \mathrm{L}, 2 \mathrm{~h}$ postprandial blood glucose $<11.1 \mathrm{mmol} / \mathrm{L}$; The diagnostic standard of hypoglycemia was blood glucose $<3 \mathrm{mmol} / \mathrm{L}$ [5]; 3) Serum C-peptide level. It includes fasting C peptide and postprandial 2hc peptide; 4) The incidence of hypoglycemia: including symptomatic hypoglycemia, severe hypoglycemia.

\subsection{Statistical analysis}

Spss20.0 statistical software was used for analysis, the measurement data was expressed with $(\bar{x} \pm s)$, the comparison was performed with $t$ test, the repeated measurement data was analyzed with ANOVA, the count data were expressed with rate (\%), the comparison was performed with chi square test, $\mathrm{P}<0.05$ was considered as statistically significant.

\section{Results}

\subsection{Comparison of blood glucose concentration between the two groups}

The blood glucose concentration of the combined treatment group increased gradually before treatment and on the 1st, 3rd and 5th day after treatment $(P<0.05)$, but there was no significant difference on the 5th, 7th and 10th day after treatment $(P>0.05)$; the blood glucose concentration of the single treatment group decreased gradually on 1D, 3D, 5D, 7d and 10d after treatment $(P<0.05)$, but the difference between the blood glucose concentration before treatment and 1D after treatment was not significant $(P>0.05)$. The blood glucose concentration of the combined treatment group was lower than that of the single treatment group on 1D, 3D and 5D after treatment $(P<$ $0.05)$, but there was no significant difference between the two groups before treatment, $7 \mathrm{~d}$ and $10 \mathrm{~d}$ after treatment $(P>0.05)$ (see Table 1).

Table 1. Comparison of blood glucose concentration between the two groups $(\bar{x} \pm s)$

\begin{tabular}{ccccc}
\hline Groups & $\mathrm{n}$ & before treatment & 1d after treatment & 3d after treatment \\
\hline Combined treatment group & 50 & $13.42 \pm 2.35$ & $12.35 \pm 2.25$ & $9.53 \pm 1.14$ \\
Single treatment group & 50 & $13.25 \pm 2.04$ & $13.72 \pm 2.36$ & $10.60 \pm 1.20$ \\
$\chi^{2}$ & & 1.886 & 4.303 & 3.366 \\
$P$ & & $>0.05$ & $<0.05$ & $<.05$ \\
\hline Table 1(Continued). Comparison of blood glucose concentration between the two groups $(\bar{x} \pm s)$ & $10 \mathrm{~d}$ after treatment \\
\hline Groups & $\mathrm{n}$ & $5 \mathrm{~d}$ after treatment & $7 \mathrm{~d}$ after treatment & $8.42 \pm 0.46$ \\
Combined treatment group & 50 & $8.35 \pm 0.65$ & $8.53 \pm 1.05$ & $8.43 \pm 0.85$ \\
$\chi^{2}$ & 50 & $9.53 \pm 1.25$ & $8.62 \pm 1.14$ & 1.638 \\
$P$ & & 3.306 & $>0.05$ & $>0.05$ \\
\hline
\end{tabular}

\subsection{Comparison of blood glucose levels between the two groups before and after treatment}

The fasting blood glucose, $2 \mathrm{~h}$ postprandial blood glucose and glycosylated hemoglobin of the two groups after treatment were lower than those before treatment $(P<0.05)$; Before treatment, there were no significant differences in fasting blood glucose, $2 \mathrm{~h}$ postprandial blood glucose and glycosylated hemoglobin between the two groups $(P>$ 0.05). After treatment, the fasting blood glucose, $2 \mathrm{~h}$ postprandial blood glucose and glycosylated hemoglobin of the combined treatment group were lower than those of the single treatment group $(P<0.05)$ (see Table 2$)$.

\subsection{Comparison of serum $C$-peptide levels between the two groups before and after treatment}

The levels of fasting C-peptide and postprandial 2-hour C-peptide in the two groups after treatment were significantly higher than those before treatment $(P<0.05)$; Before treatment, there was no significant difference in the levels of fasting $C$ peptide and postprandial 2hc peptide between the two groups $(P>0.05)$. After treatment, the 
levels of fasting $\mathrm{C}$ peptide and postprandial 2hc peptide in the combined treatment group were higher than those in the single treatment group $(P<0.05)$ (see Table 3$)$.

Table 2. Comparison of blood glucose levels between the two groups before and after treatment $(\bar{x} \pm s)$

\begin{tabular}{|c|c|c|c|c|c|}
\hline Groups & $\mathrm{n}$ & Time & $\begin{array}{l}\text { fasting blood glucose } \\
(\mathrm{mmol} / \mathrm{L})\end{array}$ & $\begin{array}{l}2 \mathrm{~h} \text { postprandial blood glucose } \\
(\mathrm{mmol} / \mathrm{L})\end{array}$ & $\begin{array}{c}\text { glycosylated hemoglo- } \\
\text { bin (\%) }\end{array}$ \\
\hline \multirow{2}{*}{$\begin{array}{l}\text { Combined treatment } \\
\text { group }\end{array}$} & \multirow{2}{*}{50} & $\begin{array}{l}\text { before treat- } \\
\text { ment }\end{array}$ & $7.63 \pm 1.25$ & $11.32 \pm 1.86$ & $10.62 \pm 1.62$ \\
\hline & & $\begin{array}{l}\text { after treat- } \\
\text { ment }\end{array}$ & $5.62 \pm 1.25$ & $8.14 \pm 1.07$ & $8.62 \pm 1.34$ \\
\hline \multirow{2}{*}{$\begin{array}{l}\text { Single treatment } \\
\text { group }\end{array}$} & \multirow{2}{*}{50} & $\begin{array}{l}\text { before treat- } \\
\text { ment }\end{array}$ & $7.66 \pm 1.43$ & $11.30 \pm 1.76$ & $10.65 \pm 1.76$ \\
\hline & & $\begin{array}{l}\text { after treat- } \\
\text { ment }\end{array}$ & $6.36 \pm 1.14$ & $9.45 \pm 1.32$ & $9.14 \pm 1.48$ \\
\hline
\end{tabular}

Table 3. Comparison of serum C-peptide levels between the two groups before and after treatment (pmol/L, $\bar{x} \pm s)$

\begin{tabular}{ccccc}
\hline Groups & $\mathrm{n}$ & Time & fasting C-peptide & postprandial 2-hour C-peptide \\
\hline Combined treatment group & 50 & before treatment & $211.14 \pm 18.86$ & $468.42 \pm 21.26$ \\
& & after treatment & $315.53 \pm 20.65$ & $790.25 \pm 20.86$ \\
Single treatment group & \multirow{2}{*}{50} & before treatment & $220.63 \pm 20.56$ & $470.25 \pm 22.64$ \\
& & after treatment & $243.15 \pm 21.43$ & $510.42 \pm 21.63$ \\
\hline
\end{tabular}

\subsection{Comparison of hypoglycemia between the two groups}

The incidence of hypoglycemia in the combined treatment group was $10.00 \%(5 / 50)$, which was lower than $52.00 \%(26 / 50)$ in the single treatment group $(P<0.05)$ (see Table 4$)$.

Table 4. Comparison of hypoglycemia between the two groups [n (\%)]

\begin{tabular}{ccccc}
\hline Groups & $\mathrm{n}$ & Symptomatic hypoglycemia & severe hypoglycemia & Total occurrence \\
\hline Combined treatment group & 50 & $5(10.00)$ & $0(0)$ & $5(10.00)$ \\
Single treatment group & 50 & $25(50.00)$ & $1(2.00)$ & $26(52.00)$ \\
$\chi^{2}$ & & & 18.550 \\
$P$ & & & $<0.05$ \\
\hline
\end{tabular}

\section{Discussion}

In clinical, for the elderly patients with high blood glucose, they often cannot eat, but their blood sugar level needs to be timely and effectively controlled, and then improve the prognosis and clinical outcome of the patients. After treatment, the blood glucose level of patients was controlled at 6.1-10.0 mmol/L [6]. In order to control the blood glucose level of patients within the effective range, insulin treatment is generally needed. However, insulin glargine is an insulin like drug that needs only once a day and can provide 24-hour glucose controlled insulin. It can simulate the secretion of basic insulin effectively, and it has the effect of continuous and no peak. In addition, it can reduce the hypoglycemia during the treatment, especially in the evening, and effectively control the blood glucose concentration of patients. There are many kinds of insulin in the market at present. But insulin glargine is a long-term insulin analogue, which is mainly produced by DNA technology by using non pathogenic strains of Escherichia coli as the production organism. Insulin of glargine is in solution state in acid environment $(\mathrm{ph}=4.0)$. After injection into subcutaneous tissue ( $\mathrm{ph}=7.4$ ), it will form precipitation and release monomer insulin slowly, which will slow down the absorption of insulin, simulate normal physiological insulin secretion for 24 hours, and then effectively and reliably supplement basic insulin. In addition, the method can be used to improve the level of insulin, it has no peak value for 24 hours, which reduces the risk of hypoglycemia in patients at night [7].

Relevant medical research results showed that the average blood glucose concentration of the treatment group on the first day, the third day and the fifth day was less than that of the control group, $P<0.05$, with statistical significance. The rest of the treatment days compared with the average blood glucose concentration after 7 days of treatment, $P>0.05$, the difference was not statistically significant [8]. Relevant medical studies also showed that 
after treatment, the average blood glucose concentrations of the experimental group and the control group at 1,3 and 5 days were $(12.11 \pm 5.1) \mathrm{mmol} / \mathrm{L},(7.6 \pm 1.2) \mathrm{mmol} / \mathrm{L},(5.5 \pm 0.9) \mathrm{mmol} / \mathrm{L}$; (thirteen point four \pm 5.7$) \mathrm{mmol} / \mathrm{L}$, $(10.8 \pm 2.0) \mathrm{mmol} / \mathrm{L},(6.6 \pm 1.0) \mathrm{mmol} / \mathrm{L}$. The difference was statistically significant $(\mathrm{P}<0.05)$. However, there was no significant difference in the average blood glucose concentration between the two groups after 7 days of treatment $(P>0.05)$ [9-10]. The results showed that the blood glucose concentration of the combined treatment group was lower than that of the single treatment group on 1D, 3D and 5D after treatment $(P<0.05)$, but there was no significant difference between the two groups before treatment, $7 \mathrm{~d}$ and $10 \mathrm{~d}$ after treatment $(P>0.05)$. After treatment, the fasting blood glucose, $2 \mathrm{~h}$ postprandial blood glucose and glycosylated hemoglobin of the combined treatment group were lower than those of the single treatment group $(P<0.05)$. After treatment, the levels of fasting C-peptide and postprandial 2H C-peptide in the combined treatment group were higher than those in the single treatment group $(P<0.05)$. The incidence of hypoglycemia in the combined treatment group was $10.00 \%(5 / 50)$, which was lower than $52.00 \%(26 / 50)$ in the single treatment group $(P<0.05)$, which was consistent with the above research results, indicating that the application of insulin glargine in elderly patients with type 2 diabetes mellitus complicated with critical medical patients can better control the blood glucose level and improve the treatment effect.

In conclusion, the clinical effect of insulin glargine in the treatment of elderly patients with type 2 diabetes mellitus complicated with critical medical patients is good, which is worthy of promotion.

\section{References}

[1] Jiang Guoying. (2018). Clinical analysis of insulin glargine in the treatment of elderly patients with type 2 diabetes mellitus complicated with critical medical patients [J]. Chinese Medical Guide, 2018, 16(9): 119-120.

[2] Han Ying. (2021). Effect analysis of saxagliptin combined with insulin glargine in the treatment of elderly patients with type 2 diabetes [J]. Diabetes Tiandi, 2021, 18(2): 78.

[3] Liu Dazheng. (2021). Effect of sitagliptin phosphate combined with insulin glargine on blood glucose control in elderly patients with type 2 diabetes [J]. Diabetes Tiandi, 2021, 18(4): 43.

[4] Wang Shuhui. (2021). Effect and safety of sitagliptin combined with insulin glargine in the treatment of elderly patients with type 2 diabetes [J]. Clinical Research and Practice, 2021, 6(5): 69-71.

[5] Zhai Fuyong. (2021). Effect of insulin glargine combined with sitagliptin in the treatment of elderly patients with type 2 diabetes mellitus [J]. Chinese Health Standard Management, 2021, 12(6): 119-122.

[6] Wang Hongyan. (2021). Effect of acarbose combined with insulin glargine on blood glucose in elderly patients with type 2 diabetes [J]. Journal of Practical Diabetes, 2021, 17(1): 129-129.

[7] Wang Shilin, Fang Xiancong, Chen Shudan, et al. (2021). Clinical observation on comprehensive assessment of elderly patients with type 2 diabetes mellitus receiving basic insulin therapy [J]. Chinese Medical Sciences, 2021, 11(1): 218-220228.

[8] Yang Chunjie, Cui Qi, Zhang Shuai, et al. (2021). Sequential treatment of newly diagnosed type 2 diabetes mellitus with intensive insulin pump [J]. Chinese Journal of Gerontology, 2021, 41(5): 941-945.

[9] Wang Lina, Guo Yuqing, Zhao Hongyan, et al. (2020). Comparison of the efficacy of insulin aspart 30 and insulin glargine combined with repaglinide in the treatment of elderly patients with newly diagnosed type 2 diabetes [J]. Chinese Primary Medicine, 2020, 27(12): 1439-1444.

[10] Tu Jingjing, Tang Jiandong, Zhang Wei, et al. (2020). Effect of dagliejing combined with insulin glargine and insulin aspart on blood glucose fluctuation in patients with poor glycemic control of type 2 diabetes [J]. Chinese Journal of Gerontology, 2020, 40(10): 2044-2047. 